\section{P2-90 HIGH LEVELS OF PLASMA HOMOCYSTEINE PREDICTS DEVELOPMENT OF HYPERTENSION IN A GENERAL POPULATION. THE TANUSHIMARU STUDY}

\section{doi:10.1136/jech.2011.142976i.25}

${ }^{1}$ A Fukami, ${ }^{2} \mathrm{H}$ Adachi, ${ }^{1} Y$ Hirai, ${ }^{1} \mathrm{M}$ Enomoto, ${ }^{1} \mathrm{M}$ Otsuka, ${ }^{1} \mathrm{Y}$ Nanjo, ${ }^{1} \mathrm{~K}$ Yoshikawa, ${ }^{1} \mathrm{E}$ Esaki, ${ }^{1} \mathrm{E}$ Kumagai, ${ }^{1} \mathrm{~K}$ Ogata, ${ }^{1} \mathrm{~A}$ Kasahara, ${ }^{1} \mathrm{E}$ Tsukagawa, ${ }^{1} \mathrm{~K}$ Yokoi, ${ }^{1} \mathrm{~K}$ Murayama, ${ }^{1} \mathrm{~T}$ Imaizumi. ${ }^{1}$ Department of Internal Medicine, Division of Cardio-Vascular Medicine, Kurume University School of Medicine, Kurume, Fukuoka, Japan; ${ }^{2}$ Department of Community Medicine, Kurume University School of Medicine, Kurume, Fukuoka, Japan

Introduction Homocysteine has been implicated in promoting atherosclerotic vascular disease. We have already demonstrated that high level of plasma homocysteine is an independent correlates for increased carotid artery wall thickness in cross-sectional study in a same cohort. The present study was designed to determine whether high plasma homocysteine levels predict the development of hypertension after ten years.

Methods A periodic epidemiological survey was performed in 1999 in Tanushimaru town. Data on fasting homocysteine were obtained from 1111 individuals (452 males, 659 females) in 1999. 512 subjects who were $\mathrm{BP} \geq 140 / 90 \mathrm{~mm} \mathrm{Hg}$ or taking hypertensive medication were excluded from the study. We enrolled 599 individuals at baseline. Ten years later, we conducted follow-up health examination in the same cohort. Of the 599 subjects, 50 had died, 49 had refused the examination. Consequently, 500 subjects (326 males, 174 females) were re-examined.

Results Of 500 normotensives ( $\mathrm{BP}<140 / 90 \mathrm{~mm} \mathrm{Hg}$ without antihypertensive medications) at baseline, 230 subjects (46\%) developed hypertension. We divided the baseline plasma homocysteine levels into tertiles. The OR for the development of hypertension after ten years was 1.66 (95\% CI 1.02 to 2.73 ) in the highest tertile vs the lowest tertile of homocysteine level after adjustment for confounding factors.

Conclusion High levels of homocysteine predicted the development of hypertension in normotensive subjects. We may propose measurement of plasma homocysteine as a predictive marker for hypertension in clinical practice.

\section{P2-91 CALCIUM, PHOSPHORUS, AND CARBOHYDRATES AND THE RISK OF COLORECTAL CANCER IN POLAND}

\section{doi:10.1136/jech.2011.142976i.26}

${ }^{1} \mathrm{~A}$ Galas, ${ }^{*}{ }^{1} \mathrm{E}$ Sochacka-Tatara, ${ }^{1} \mathrm{~A}$ Pac, ${ }^{2} \mathrm{~K}$ Zawisza, ${ }^{3} \mathrm{~J}$ Kulig, ${ }^{1} \mathrm{~W}$ Jedrychowski. ${ }^{1}$ Jagiellonian University - Medical College, Chair of Epidemiology and Preventive Medicine, Department of Epidemiology, Krakow, Poland; ' Jagiellonian University Medical College, Chair of Epidemiology and Preventive Medicine, Department of Sociology, Krakow, Poland; ${ }^{3}$ Jagiellonian University - Medical College, I Chair of General Surgery and Department of Gastroenterological Surgery, Krakow, Poland

Introduction An increasing trends of colorectal (CRC) morbidity and mortality across developed countries is observed. Epidemiological studies showed that majority of those cases are due to improper diet. The results on colorectal risk modification by dietary factors in central Europe are scarce. The objective of the present study was to evaluate the role of calcium, phosphorus and polysaccharides intake on colorectal cancer risk in Poland.

Methods The hospital-based case-control study was carried out in Poland in 2000-2010. In total, 637 histologically confirmed cancer cases and 874 controls were recruited. Information about frequency and quantity of consumption of 148 alimentary items and numerous confounders was gathered by questionnaire.

Results After adjustment, increase consumption of calcium was associated with decreased risk of colorectal cancer ( $\mathrm{OR}=0.59,95 \%$ CI 0.41 to 0.86). Similar change in risk was observed with increased consumption of phosphorus ( $\mathrm{OR}=0.58,95 \% \mathrm{CI} 0.44$ to 0.75 ). On the other hand, an increasing risk of CRC with increasing consumption of polysaccharides was observed (in quartiles: ORs: $1.20,1.39,1.56$, respectively).

Conclusions The results of the present study support the protective role of high intake of calcium and phosphorus in the aetiology of colorectal cancer. Additionally they suggest that high consumption of polysaccharides may lead to elevated risk of CRC.

\section{P2-92 HORMONE MARKERS AND ASTHMA IN YOUNG ADULTHOOD: RESULTS FROM A HISTORICAL COHORT}

doi:10.1136/jech.2011.142976i.27

B Galobardes, * S Patel, J Henderson, M Jeffreys, G D Smith. CAiTE, School of Social and Community Medicine, University of Bristol, Bristol, UK

Introduction Prevalence of asthma is higher in boys during childhood but in women in adulthood. Recent studies point to the role of hormones in explaining these gender-age differences but often single markers of hormone levels have been assessed. The aim of this study is to investigate the association of history of asthma in young adulthood with different hormone markers during adolescence.

Methods Historical cohort of students (11274 men and 3502 women) attending Glasgow University between 1948 and 1968, that participated to a health survey. Medical history of asthma, eczema/urticaria and hay fever were collected. Age of menarche, duration and regularity of menstruation and acne were also recorded.

Results Among women, older age of menarche (1-year increase) was associated with atopic asthma (asthma with eczema/urticaria or hay fever) ( $\mathrm{OR}=1.39,95 \% \mathrm{CI} 1.05$ to 1.85$)$. Irregular menstrual cycle was associated with atopic asthma (OR $=2.68,95 \%$ CI 1.28 to 5.61$)$ and atopy (eczema /urticaria or hay fever) (OR $=1.39,95 \%$ CI 1.05 to 1.83 ) but not with non-atopic asthma ( $\mathrm{OR}=0.86,95 \% \mathrm{CI} 0.36$ to $2.05)$. Acne was not robustly associated with atopic $(\mathrm{OR}=0.32,95 \%$ CI 0.08 to 1.32 ) or non-atopic ( $\mathrm{OR}=0.78,0.33$ to 1.86 ) asthma. Among men, those with acne had lower risk of eczema/urticaria alone $(\mathrm{OR}=0.44,95 \% \mathrm{CI} 0.29$ to 0.68$)$ but some evidence of higher risk of hay fever alone ( $\mathrm{OR}=1.23,95 \%$ CI 0.98 to 1.53$)$.

Conclusion Age of menarche and irregular menstruation were associated with higher risk of atopic asthma and atopy in women whereas acne was associated with lower risk of eczema/urticaria among male students.

\section{P2-93 MORTALITY FROM CHRONIC DISEASES IN BRAZIL, 1980-2008}

doi:10.1136/jech.2011.142976i.28

${ }^{1} \mathrm{~L}$ Garcia, ${ }^{*} \mathrm{M}$ Montenegro, ${ }^{3} \mathrm{~W}$ Ramalho. ${ }^{1}$ Institute for Applied Economic Research IPEA, Brasilia, Brazil; ${ }^{2}$ Brazilian Ministry of Health, Brasilia, Brazil; ${ }^{3}$ University of Brasilia, Brasilia, Brazil

Introduction The profile of causes of death in Brazil has changed in recent decades. The objective is to describe the evolution of mortality from chronic diseases in Brazil, 1980-2008.

Methods Crude and age- and sex-standardised mortality rates (SMR) and proportional mortality ratios (PMR) were calculated from data from the Mortality Information System of the Ministry of Health. Results In the period of 1980-2008, diseases of the circulatory system were the main cause of death $(\mathrm{PMR}=32.1 \%$ in $1980,31.8 \%$ in 2008). Despite the stability of this indicator for the whole country, there were significant regional differences. Neoplasms were the fourth group of causes of death in 1980 (PMR=10.4), and the second in 2008 ( $\mathrm{PMR}=16.8 \%$ ), with a positive percentage change of $62.1 \%$. This trend of strong growth was seen in all 